\title{
Interactions between estrogenic chemicals in binary mixtures investigated using vitellogenin induction and factorial analysis
}

\author{
Liwei Sun ${ }^{\mathrm{a}, \mathrm{b}}$, Jinmiao Zha ${ }^{\mathrm{b}}$, Zijian Wang ${ }^{\mathrm{b}, *}$ \\ ${ }^{a}$ College of Biological and Environmental Engineering, Zhejiang University of Technology, No.18 Chaowang Road, Hangzhou 310032, PR China \\ ${ }^{\mathrm{b}}$ State Key Laboratory of Environmental Aquatic Chemistry, Research Center for Eco-Environmental Sciences, Chinese Academy of Sciences, Shuangqing Rd. 18, Haidian District, \\ P.O. Box 2871, Beijing 100085, PR China
}

\section{A R T I C L E I N F O}

\section{Article history:}

Received 17 September 2008

Received in revised form 24 November 2008

Accepted 30 November 2008

Available online 10 January 2009

\section{Keywords:}

Statistical design

Concentration addition

Mixture effects

Japanese medaka

\begin{abstract}
A B S T R A C T
Considerable progress has been made in assessing the combined effects of chemicals, but the effect of mixtures remains one of the most daunting challenges in environmental toxicology. In this study, the effects of binary mixtures of estrogenic chemicals were investigated using plasma vitellogenin (VTG) induction in male adult Japanese medaka (Oryzias latipes) as the endpoint. We focused on whether or not the factorial design using rigorous statistical methods was appropriate for the identification of possible interactions. The estrogenic activities of $17 \beta$-estradiol (E2), 4-tert-nonylphenol (NP) and bisphenol A (BPA) were investigated in medaka following $14 \mathrm{~d}$ of exposure. Although all chemicals induced concentration-dependent increases in plasma VTG, a complete concentration-response curve was obtained only for E2, while partial curves were obtained for NP and BPA. Therefore, a $3 \times 3$ factorial design was employed to identify both the individual and interactive effects. A significant difference in mean plasma VTG induction was found when any chemical was considered alone. However, no significant interactions between E2 and NP, E2 and BPA, and NP and BPA were demonstrated. These results suggest that these three model estrogenic chemicals share a common mechanism for inducing VTG synthesis, and that no interactions occur when they act in combination. We also demonstrated the ability of this experimental design to detect interactions between binary mixtures, which will allow the assessment of biological effects of more complex mixtures in future research.
\end{abstract}

(c) 2008 Elsevier Ltd. All rights reserved.

\section{Introduction}

In the last two decades, increased attention has been paid to synthetic and natural chemicals that have the potential to interfere with the endocrine systems of organisms (Matthiessen, 2003; Sullivan and Spence, 2003). Extensive evidence now exists to show that these substances, termed endocrine disrupting chemicals (EDCs), may be responsible for adverse reproductive and developmental effects in both wildlife and humans (OECD, 1997; US EPA, 2002). Since the aquatic environment is the ultimate sink for most environmental pollutants, most cases of endocrine disruption and associated reproductive impairment in wildlife have been reported in aquatic organisms, particularly in various species of fish (Tyler et al., 1998; Sheahan et al., 2002).

The globally increased interest in EDCs in aquatic ecosystems has resulted in various research and monitoring programs which have demonstrated that a wide range of estrogenic chemicals are sometimes detected simultaneously (Desbrow et al., 1998; Staples et al., 2000; Petrovic et al., 2004). Even though estrogenic chemi-

\footnotetext{
* Corresponding author. Tel./fax: +86 1062849140.

E-mail address: wangzj@rcees.ac.cn (Z. Wang).
}

cals tend to be present at low concentrations and exhibit weak activity individually, the potential exposure to mixtures of these chemicals suggests the possibility of additive and/or interactive effects, which could produce a significant combined response (Payne et al., 2000; Thorpe et al., 2001). In toxicology, depending on the presumed modes of action of the mixture's components, two concepts are used to predict the effects of mixtures: concentration addition (CA) and independent action (IA). The effects of the mixture can be additive, but if the observed responses are stronger or weaker than expected, then the combined effect is described as being either synergistic or antagonistic, respectively( US EPA, 2000). Considerable evidence suggests that the effects of estrogenic mixtures can be predicted successfully by CA (both in vitro and in vivo, reviewed in Kortenkamp, 2007). In fact, the CA model has been suggested as the pragmatic default approach when calculating the quantitative effects of any combination of agents, irrespective of the (dis)similarity of its components (de Zwart and Posthuma, 2005). However, due to the weakness of the mathematical formula and the varying natures of the chemicals tested, no concept currently provides a "general solution" for assessing the interactions between estrogenic chemicals (Thorpe et al., 2001; Altenburger et al., 2004; Backhaus et al., 2004). 
Vitellogenin (VTG) is normally synthesized in mature female fish under the control of estrogen, mediated by estrogen receptors (ERs), and is then incorporated into growing oocytes. However, since both sexes carry ERs and the VTG gene, males or juveniles can be induced to synthesize VTG after exposure to estrogen or estrogen-mimics (Denslow et al., 1999; Brion et al., 2002). Thus, the presence of plasma VTG in male or juvenile fish has been recognized as a sensitive biomarker of estrogenic exposure (Sumpter and Jobling, 1995). Additionally, VTG is a robust indicator of estrogen exposure compared with other endpoints, such as reproductive performance (Brian et al., 2007; Jukosky et al., 2008).

Although there has been increased research into the interactions of estrogenic chemicals in vivo (Thorpe et al., 2001, 2003; Brian et al., 2005; Lin and Janz, 2006; Correia et al., 2007), these studies are still limited compared with in vitro studies, due to the biological complexity of the organisms and the practical limitations of simultaneously conducting large-scale experiments (Thorpe et al., 2003). The limitation of the mathematical modeling procedure involved hampered this progress as well. In this study, the estrogenic activities of three estrogenic chemicals, namely $17 \beta$-estradiol (2) 4-tert-nonylphenol (NP) and bisphenol A (BPA), were investigated both individually and in binary mixtures, by measuring plasma VTG induction in male adult Japanese medaka (Oryzias latipes), using rigorous statistical methods. This study aimed to determine whether or not the factorial design was appropriate for the identification of possible interactions of estrogenic chemical, and if this method could therefore be applied to other environmentally relevant mixtures and to provide information on the nature of chemical interactions.

\section{Materials and methods}

\subsection{Test chemicals}

Three estrogenic chemicals, including the natural steroidal estrogen E2, and the estrogen-mimicking compounds NP and $\mathrm{BPA}$, were selected for study because of their presence in the aquatic environment and their potential to produce reproductive abnormalities in wild fish populations. E2 (98.0\% purity), NP $(99.0 \%$ purity) and BPA (98.0\% purity) were all purchased from Sigma-Aldrich, USA. All other chemicals were analytical or HPLC grade.

\subsection{Test fish}

Japanese medaka (d-rR) stock originated from the Laboratory of Freshwater Fish at the Bioscience Center of Nagoya University, Japan. The stock has been maintained and propagated in our laboratory for more than 5 years. Medaka were kept in charcoaldechlorinated tap water at a constant temperature $\left(25 \pm 1^{\circ} \mathrm{C}\right)$, with a 16:8 h (light: dark) photoperiod. The brood stock was fed three times daily, once with brine shrimp (Artemia nauplii) and twice with commercial fish food (TetraMin).

\subsection{Experimental design}

At the beginning of the experiment, male adult medaka (approximately 5 months old) were acclimated for 1 week to experimental test conditions. Exposure studies were conducted in $15 \mathrm{~L}$ glass aquaria under flow-through system conditions. Twelve fish were randomly assigned to each aquarium and duplicate aquaria were used for each exposure level. Stock solutions were prepared weekly by dissolving the chemicals in HPLC-grade acetone (Fisher Scientific, USA). Solutions were delivered into the experimental tanks using a dilution apparatus equipped with a multi-channel peristaltic pump and mixing chambers, which sup- plied a constant flow equivalent to seven times the aquaria volume per day. Dissolved oxygen $(>80 \%)$, water temperature $\left(25 \pm 1^{\circ} \mathrm{C}\right)$, and the functioning of the delivery system were monitored throughout the study.

The test duration was $14 \mathrm{~d}$ and the feeding regime was maintained throughout the exposure period. The aquaria were cleaned once a week as the formation of micelles might disturb the fish, particularly in the high-exposure groups.

\subsubsection{Estrogenicity testing of single compounds (study I)}

Based on the results of an initial range-finding study (data not shown) and the solubility of each test chemical, the fish were exposed to nominal E2 concentrations of 5, 25, 125, 625, and $3125 \mathrm{ng} \mathrm{L}^{-1}$, NP of $10,50,250$, and $1250 \mu \mathrm{g} \mathrm{L}^{-1}$, and BPA of 20 , 100,500 , and $2500 \mu \mathrm{g} \mathrm{L}^{-1}$. In addition, the dilution water control (DWC) and solvent control (SC) containing $0.1 \mathrm{~mL} \mathrm{~L}^{-1}$ acetone were run to provide baseline data.

\subsubsection{Estrogenicity testing of the mixture (study II)}

Based on the results of study I, a factorial design, which allows the effects of individual chemicals and their interactions to be statistically tested, was employed. Three distinct $3 \times 3$ factorial designs including E2 $\times \mathrm{NP}$, E2 $\times \mathrm{BPA}$, and $\mathrm{NP} \times \mathrm{BPA}$ were crossed for a total of 27 treatment combinations. The dose levels were chosen based on the concentration-responses of the individual chemicals determined in study I. The nominal concentrations of the three chemicals were: E2 at 5, 25, and $125 \mathrm{ng} \mathrm{L}^{-1}$, NP at 10,50 , and $250 \mu \mathrm{g} \mathrm{L}^{-1}$, and BPA at 100,500 , and $2500 \mu \mathrm{g} \mathrm{L}^{-1}$. The DWC and SC were also included.

\subsection{Analytical chemistry}

The concentrations of chemicals were monitored throughout the study. Water samples were collected from each tank in solvent-rinsed glass bottles on days 0,7 and 14 of the exposures. The analysis was performed according to the methods of Quintana et al. (2004) and Zhou (2008). Briefly, filtered water samples were spiked with deuterated standards, and extracted using preconditioned solid-phase extraction (SPE) cartridges. E2 was eluted in methanol/tert-butyl methyl ether $(1 / 9 ; \mathrm{v} / \mathrm{v})$ and derivatized by N,O-bis-(trimethylsilyl)-trifluoroacetamide (BSTFA) $+1 \%$ trimethylchlorosilane (TMCS) $+0.5 \%$ trimethylsilylimidazole (TMSI), while NP and BPA were eluted in methanol/dichloromethane $(1 / 9 ; \mathrm{v} / \mathrm{v})$ and derivatized by BSTFA $+1 \%$ TMCS. The samples were analyzed using a 6890 gas chromatograph/5973 mass spectrometer (Agilent, USA). The detection limits for E2, NP and BPA were 0.03, 0.2 and $0.1 \mathrm{ng} \mathrm{L}^{-1}$, respectively. The mean measured concentrations during the exposure period ranged from $81 \%$ to $102 \%, 85 \%$ to $99 \%$ and $85 \%$ to $103 \%$ of the nominal values for E2, NP and BPA, respectively. Since good agreement existed between the nominal and actual exposure concentrations, subsequent analyses of biological effects were based on nominal concentrations.

\subsection{Blood sampling and measurement of plasma vitellogenin}

After each exposure, fish were sacrificed by keeping them on ice for 1-2 min, and the blood samples were collected in heparinized microcapillary tubes by severing the caudal peduncle. The volume was measured, and then the sampling buffer $(20 \mathrm{mM}$ Tris, $1 \mathrm{mM}$ EDTA, $150 \mathrm{mM} \mathrm{NaCl}$, and $25 \mathrm{KIU} \mathrm{mL}^{-1}$ aprotinin at $\mathrm{pH} 7.5$ ) was added individually. The samples were immediately centrifuged ( $8000 \mathrm{~g}, 10 \mathrm{~min}, 4^{\circ} \mathrm{C}$ ), and the plasma was stored at $-80^{\circ} \mathrm{C}$ until analysis.

Plasma VTG levels were measured using a competitive enzymelinked immunosorbent assay (ELISA). The procedure was performed based on the method described by Brion et al. (2002). 
The working range of the assay was from 30 to $1500 \mathrm{ng} \mathrm{mL}^{-1}$ (20\%-80\% binding), and the minimal detection limit was $15 \mathrm{ng} \mathrm{mL}^{-1}$ for purified VTG. To avoid matrix effect, a dilution of at least 1:100 was chosen for the routine assay. The intra- and inter-assay variations at $50 \%$ binding were $5.6 \%$ and $10.2 \%$, respectively. Purified medaka VTG was used as a standard, and VTG in diluted samples was measured in triplicate.

\subsection{Statistical analysis}

When VTG value was not detected in male fish, we used the detection limit as the VTG value. Statistical concentration-response analyses were performed for all compounds by applying a best fit procedure (Scholze et al., 2001). Interactions in binary mixtures were examined using a full factorial analysis of variance (ANOVA) experimental design, and profile plots (interaction plots) were used for comparing marginal means. For two factors, parallel lines indicate no interaction between the factors (i.e. the two chemicals interact in an additive manner), whereas nonparallel lines indicate a nonadditive interaction. All statistical analyses were performed using SPSS 13.0 (SPSS, Chicago, IL, USA) and Origin 7.0 (OriginLab, Northampton, MA, USA). The critical value for statistical significance was $p \leqslant 0.05$.

\section{Results}

\subsection{Estrogenicity of E2, NP and BPA}

No mortalities were observed in fish exposed to any of the test chemicals in any of the experiments, indicating that the chemicals tested were not acutely toxic at the tested concentrations and that the fish were not unduly stressed. The concentrations of plasma VTG were below the detection limit in both the DWC and the SC fish. E2 induced plasma VTG in a concentration-dependent manner. The Logic regression model was selected since the value of $R^{2}$ (coefficient of determination) was greater than 0.999. Fig. 1 showed the concentration-response data for E2 and its estimated full regression curve. The estimated EC50 value was $225 \mathrm{ng} \mathrm{L}^{-1}$, which was in close agreement with values from previous research (21-d EC50 value was $200 \mathrm{ng} \mathrm{L}^{-1}$; Thompson et al., 2000).

In contrast, due to the relatively low estrogenic activity and limited solubility of NP and BPA, we could not reach the maximum VTG induction plateaus which resulted in no nonlinear regression models fitted to the experimental data well. Partial, but not full, concentration-response relationships of NP and BPA are given in Fig. 2.

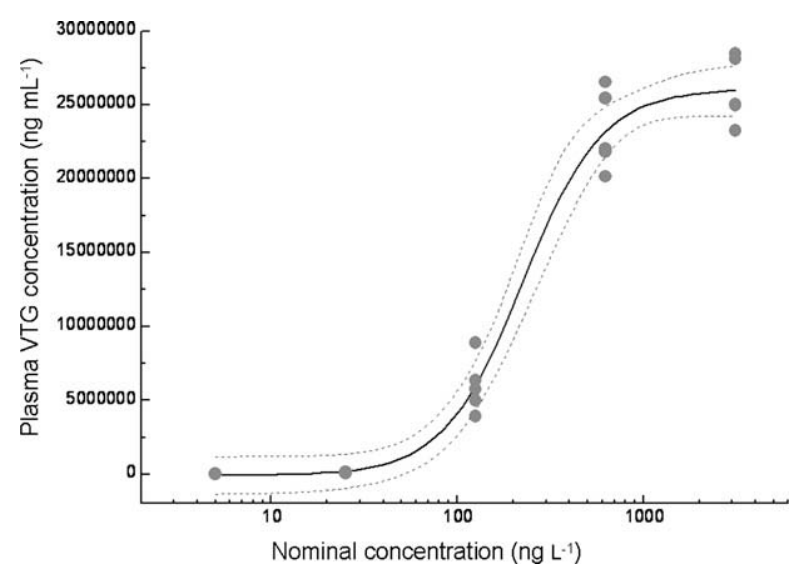

Fig. 1. Plasma vitellogenin (VTG) concentrations in male adult medaka exposed to E2 for $14 \mathrm{~d}$. The concentration-response was estimated using a Logit regression model which is shown as a solid line with $95 \%$ confidence interval.

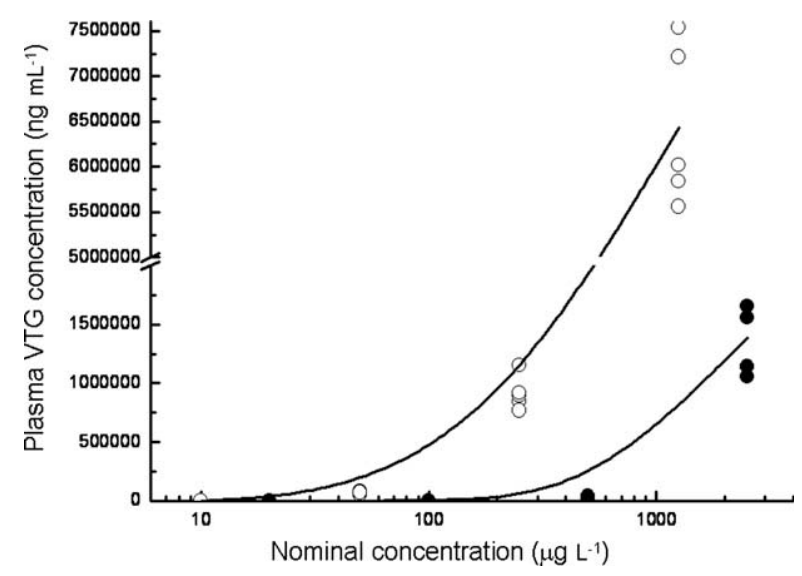

Fig. 2. Plasma vitellogenin (VTG) concentrations in male adult medaka exposed to NP (open circles) and BPA (closed circles) for $14 \mathrm{~d}$.

\subsection{Interactions in binary mixtures}

No mortalities were observed in any of the treatment groups and the concentrations of plasma VTG were below the detection limit in both the DWC and the SC fish. The VTG concentrations in adult male fish exposed to binary mixtures are shown in Fig. 3. Potential interactions in the three distinct binary mixtures were examined by using a full factorial ANOVA. Results of $3 \times 3$ factorial ANOVA are presented in Table 1 . A statistically significant difference $(p<0.01)$ in mean values of plasma VTG induction was found when any chemical was considered alone. However, no significant interactions were demonstrated between E2 and NP, E2 and BPA, and NP and BPA $(p>0.05)$. Additionally, the interaction effects were confirmed visually by the profile plots (Fig. 4). Profile plots produced approximately parallel lines, also suggesting no interactions between E2 and NP, E2 and BPA, or BPA and NP.

\section{Discussion}

Good evidence has become available to show that most estrogenic chemicals do not necessarily share a structural relationship with natural estrogens, such as E2, but still act as ER agonists (Witorsch, 2002). Compared with E2, the estrogenic potency of most of these chemicals is low, since their affinity for ERs is much lower. Yamaguchi et al. (2005) investigated the expression of estrogenresponsive genes in the liver of male medaka after $8 \mathrm{~h}$ exposure, and the relative estrogenic potencies of the tested chemicals decreased in the order of E2 $(100)>\mathrm{NP}(0.02)>\mathrm{BPA}(0.001)$. The findings of Seki et al. (2003) suggested that the relative in vivo estrogenic activity of NP was $1 / 3580$ that of E2, as determined by assessing the effects on sexual differentiation and hepatic VTG induction in medaka. The results of the present study are in accordance with these previous studies. However, the biologic effects of estrogenic chemicals cannot be dismissed as insignificant solely on the basis of their low potency compared with steroidal estrogens. Even sub-NOEC (no observed effect concentration) levels of estrogenic chemicals may have effects when combined with similarlyacting chemicals (Rajapakse et al., 2002; Silva et al., 2002).

Regarding the in vivo assessment of the toxicity of estrogenic chemicals mixtures in fish using VTG induction as the endpoint, Thorpe et al. (2001) were the first to study the effects of binary mixtures, and the results suggested that NP acted via the same mechanism as E2 in inducing VTG in juvenile rainbow trout (Oncorhynchus mykiss), while the pesticide methoxychlor possibly acted via a different mechanism. The E2 and EE2 in the binary mixture also trigger VTG synthesis similarly (Thorpe et al., 2003). Brian 

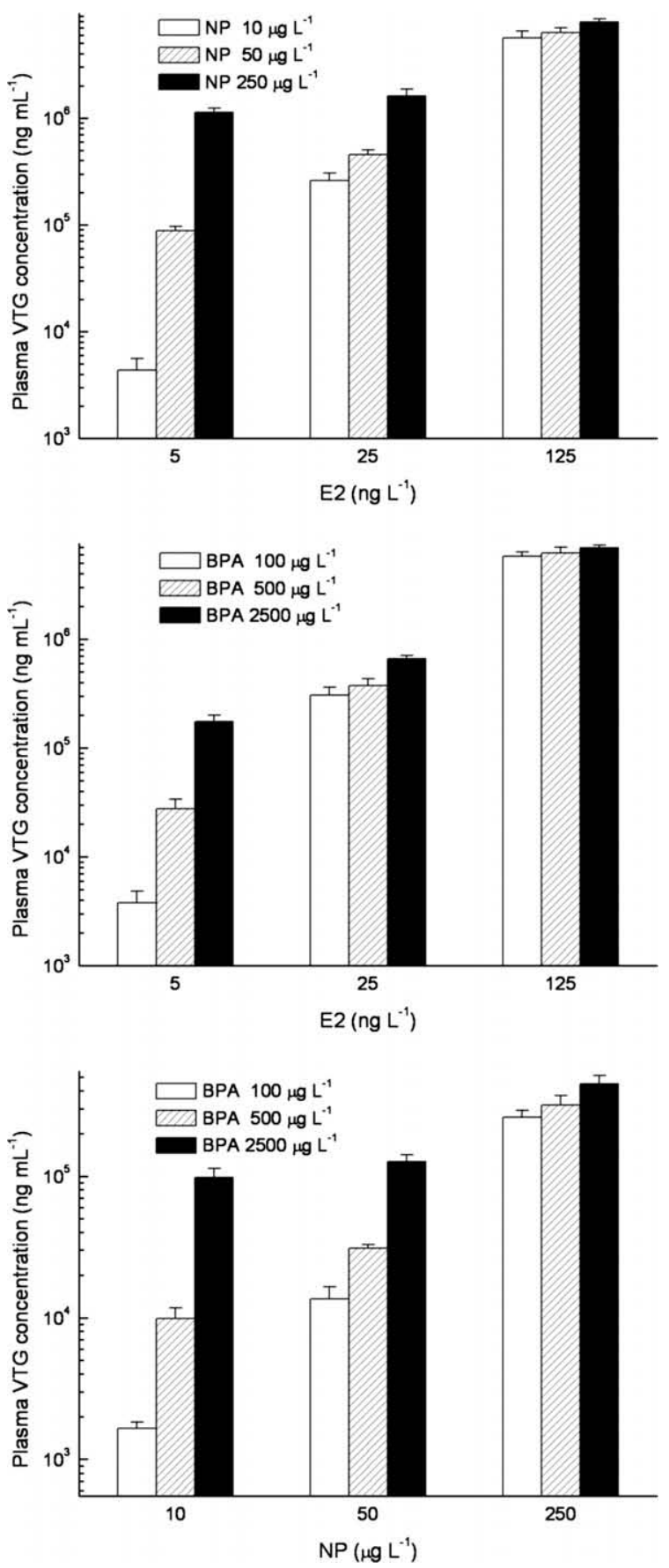

Fig. 3. Plasma vitellogenin (VTG) concentrations in male adult medaka exposed to the binary mixtures for $14 \mathrm{~d}$, using the $3 \times 3$ factorial design. Values are shown as mean VTG concentrations, and error bars represent the standard deviation.

Table 1

Results of $3 \times 3$ factorial analysis of variance (ANOVA).

\begin{tabular}{llrr}
\hline Mixture & Source & \multicolumn{1}{c}{ F } & \multicolumn{1}{c}{$p$} \\
\hline E2 $\times$ NP & E2 & 775.600 & $<0.001$ \\
& NP & 45.351 & $<0.001$ \\
E2 $\times$ BPA & E2 NP & 2.059 & 0.114 \\
& BPA & 1125.783 & $<0.001$ \\
& E2 $\times$ BPA & 7.744 & 0.002 \\
NP $\times$ BPA & NP & 2.188 & 0.097 \\
& BPA & 364.717 & $<0.001$ \\
& NP $\times$ BPA & 60.595 & $<0.001$ \\
& & 2.421 & 0.073 \\
\hline
\end{tabular}
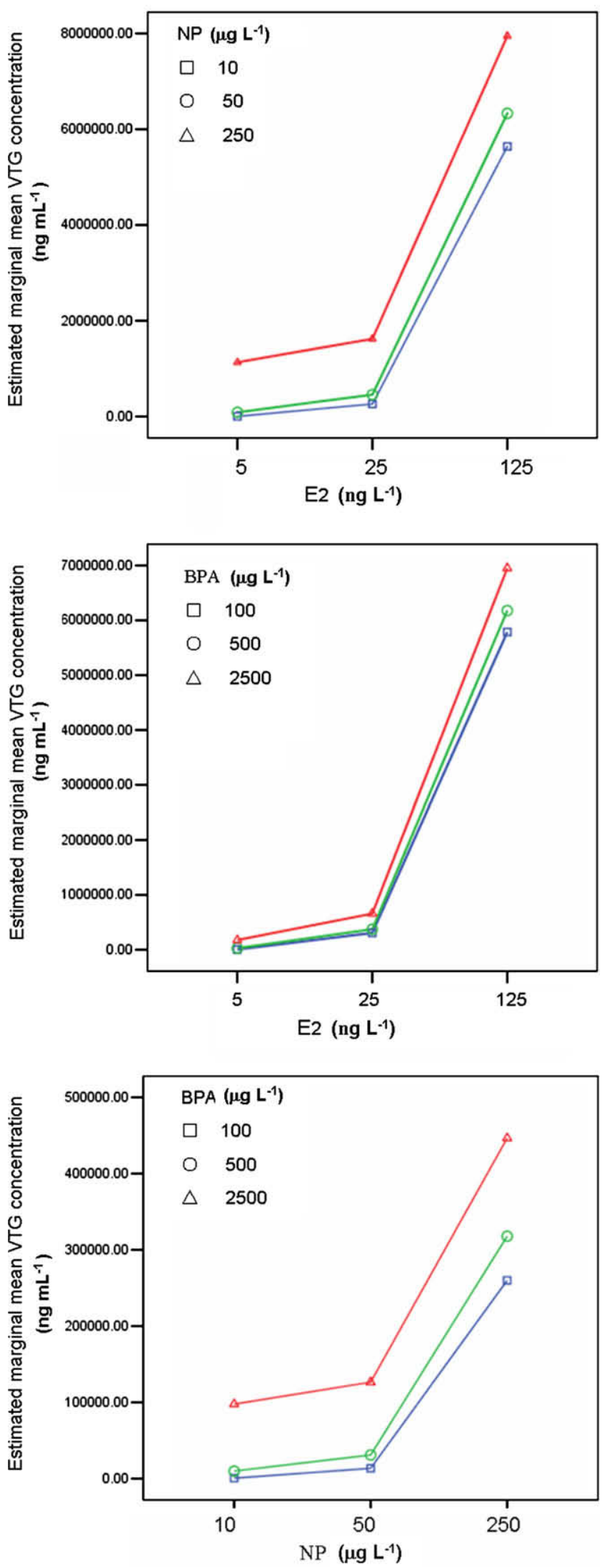

Fig. 4. Interactions between the individual chemicals illustrated by profile plots (interaction plots) with comparative marginal means of vitellogenin (VTG) induction. 
et al. (2005) analyzed the combined effects of a multicomponent mixture of five estrogenic chemicals, including E2, EE2, NP, octylphenol (OP), and BPA, and the results demonstrated that estrogenic chemicals have the capacity to act together in an additive manner. A similar conclusion was reached in juvenile sea bass (Dicentrarchus labrax) when assessing the combined effects of estrogenic chemicals in marine ecosystems (Correia et al., 2007). Our present work also found E2, NP and BPA act via the same mechanism in mediating the vitellogenic response, and no interactions occur between E2 and NP, E2 and BPA, or NP and BPA, which was in agreement with these earlier reports.

In all these earlier reports, the CA model was applied successfully to identify whether or not the mechanisms of action of chemicals in the combinations are similar. However, as mentioned above, no model can provide a "general solution" for assessing the combination effects of chemicals, as well as the CA model. Jukosky et al. (2008) exposed pairs of medaka to mixtures of NP, $\mathrm{EE} 2$, and E2, and found that mixtures elicited lower vitellogenic induction than equipotent concentrations of E2 alone. They therefore concluded that simple additive mixture models (i.e. the CA model) may not predict all relevant physiological responses. In addition, the CA model makes some implicit assumptions which restrict its application inevitability .For instance, the concentration-effect relationship of the individual component of the mixture estimated by a nonlinear regression model should be monotonic (Scholze et al., 2001), and the models are defined only for positive fractional effects. As a consequence, the models are not suitable for describing phenomena such as stimulating effects at low concentrations (i.e. hormesis) or the occurrence of thresholds (Backhaus et al., 2004). Moreover, as shown in the present work, when the maximal plateau of VTG induction cannot be obtained and no appropriate regression analysis can therefore be fitted to the data, the application of the CA model is limited. A further problem arises for mathematical reasons: when considering components with different asymptotical maximal effects, it is not possible to calculate the mixture concentrations for all effects between (and above) these two individual maximal effects using the CA model (Thorpe et al., 2001; Silva et al., 2002). In the present work, even if we could obtain maximal plateau values of VTG induction for NP and BPA, the maximal effects would probably be different: different chemicals often produce different maximal responses, as shown by Thorpe et al. (2001). It is also important to recognize that the CA model can only be applied when the mixture is completely defined in terms of the number of chemicals present as well as the mixture ratio (Brian et al., 2005). A mixture analysis based on the so-called fixed-ratio design can be problematic, since the concentration of chemicals can show pronounced temporal and spatial variation in the environment.

In the present study, ANOVA supported by factorial design was used to test for the combined effects of estrogenic chemicals, and the result was in line with the previous reports using the CA model. Similar experimental designs have been applied successfully to testing the in vivo interactions between individual chemicals not only in fish (Lin and Janz, 2006), but also in amphibians (Sullivan and Spence, 2003). This statistical experimental approach does not require dose-effect equations for each individual chemical of the mixture per se (Feron and Groten, 2002), and can avoid most of the limitations of the CA model. However, when assessing the effects of more complex mixtures, the experimental procedures, especially for in vivo studies, mean that, for both humane and practical reasons, researchers are forced to reduce the number of experimental groups. A number of statistical designs that are more efficient and cost-effective are therefore available, such as ray designs, central or simplex composite designs, and fractional factorial designs, or simultaneity with response surface analysis (Charles et al., 2002; Feron and Groten, 2002). Careful choice of experimen- tal design is a prerequisite for a successful mixture toxicity study. However, it should be noted that these statistical designs are more useful for interpretation than for prediction. Collaboration between experimental toxicologists, biomathematicians, pharmacologists and model developers is required to ensure parallel and cocoordinated research in this challenging area of mixture toxicology (Feron and Groten, 2002).

\section{Conclusion}

The present study assessed the in vivo potency of binary mixtures of estrogenic chemicals, including E2, NP and BPA, using plasma VTG induction in adult male Japanese medaka as a measure of effect. We focused on whether or not the factorial design using rigorous statistical methods was appropriate for the identification of possible interactions. Although the synthesis of VTG was markedly increased following treatment with estrogenic chemicals, no significant interactions between the components of the mixtures were found. These results were consistent with previous reports, and demonstrated the ability of this experimental design to detect interactions between components of binary mixtures, so allowing for the assessment of the biological effects of environmentally relevant mixtures involving more complex chemicals.

\section{Acknowledgements}

We gratefully acknowledge the supports of National Basic Research Program of China (2007CB407304) and Natural Science Foundation of China $(20737003,20621703)$ for conducting this research.

\section{References}

Altenburger, R., Walter, H., Grote, M., 2004. What contributes to the combined effect of a complex mixture? Environ. Sci. Technol. 38, 6353-6362.

Backhaus, T., Arrhenius, A., Blanck, H., 2004. Toxicity of a mixture of dissimilarly acting substances to natural algal communities: predictive power and limitations of independent action and concentration addition. Environ. Sci. Technol. 38, 6363-6370.

Brian, J.V., Harris, C.A., Scholze, M., Backhaus, T., Booy, P., Lamoree, M., Pojana, G., Jonkers, N., Runnalls, T., Bonfà, A., Marcomini, A., Sumpter, J.P., 2005. Accurate prediction of the response of freshwater fish to a mixture of estrogenic chemicals. Environ. Health Perspect. 113, 721-728.

Brian, J.V., Harris, C.A., Scholze, M., Kortenkamp, A., Booy, P., Lamoree, M., Pojana, G. Jonkers, N., Marcomini, A., Sumpter, J.P., 2007. Evidence of estrogenic mixture effects on the reproductive performance of fish. Environ. Sci. Technol. 41, 337344.

Brion, F., Nilsen, B.M., Eidem, J.K., Goksoyr, A., Porcher, J.M., 2002. Development and validation of an enzyme-linked immunosorbent assay to measure vitellogenin in the zebrafish (Danio rerio). Environ. Toxicol. Chem. 21, 1699-1708.

Charles, G.D., Gennings, C., Zacharewski, T.R., Gollapudi, B.B., Carney, E.W., 2002. An approach for assessing estrogen receptor-mediated interactions in mixtures of three chemicals: a pilot study. Toxicol. Sci. 68, 349-360.

Correia, A.D., Freitas, S., Scholze, M.F., Gonçalves, J., Booij, P., Lamoree, M.H., Mañanós, E., Reis-Henriques, M.A., 2007. Mixtures of estrogenic chemicals enhance vitellogenic response in sea bass. Environ. Health Perspect. 115, 115121.

de Zwart, D., Posthuma, L., 2005. Complex mixture toxicity for single and multiple species: proposed methodologies. Environ. Toxicol. Chem. 24, 26652676.

Denslow, N.D., Chow, M.C., Kroll, K.J., Green, L., 1999. Vitellogenin as a biomarker of exposure for estrogen or estrogen mimics. Ecotoxicology 8, 385-398.

Desbrow, C., Routledge, E.J., Brighty, G.C., Sumpter, J.P., Waldock, M., 1998. Identification of estrogenic chemicals in STW effluent: 1. Chemical fractionation and in vitro biological screening. Environ. Sci. Technol. 32, 1549-1558.

Feron, V.J., Groten, J.P., 2002. Toxicological evaluation of chemical mixtures. Food Chem. Toxicol. 40, 825-839.

Jukosky, J.A., Watzin, M.C., Leiter, J.C., 2008. The effects of environmentally relevant mixtures of estrogens on Japanese medaka (Oryzias latipes) reproduction. Aquat. Toxicol. 86, 323-331.

Kortenkamp, A., 2007. Ten years of mixing cocktails: a review of combination effects of endocrine-disrupting chemicals. Environ. Health Perspect. 115, 98105.

Lin, L.L., Janz, D.M., 2006. Effects of binary mixtures of xenoestrogens on gonadal development and reproduction in zebrafish. Aquat. Toxicol. 80, 382-395. 
Matthiessen, P., 2003. Historical perspective on endocrine disruption in wildlife Pure Appl. Chem. 75, 2197-2206.

OECD, 1997. Draft detailed review paper: appraisal of test methods for sexhormone disrupting chemicals. Paris, France.

Payne, J., Rajapakse, N., Wilkins, M., Kortenkamp, A., 2000. Prediction and assessment of the effects of mixtures of four xenoestrogens. Environ. Health Perspect. 108, 983-987.

Petrovic, M., Eljarrat, E., Lopez de Alda, M.J., Barceló, D., 2004. Endocrine disrupting compounds and other emerging contaminants in the environment: a survey on new monitoring strategies and occurrence data. Anal. Bioanal. Chem. 378, 549562.

Quintana, J.B., Carpinteiro, J., Rodríguez, I., Lorenzo, R.A., Carro, A.M., Cela, R., 2004 Determination of natural and synthetic estrogens in water by gas chromatography with mass spectrometric detection. J. Chromatogr. A 1024 177-185.

Rajapakse, N., Silva, E., Kortenkamp, A., 2002. Combining xenoestrogens at levels below individual no-observed-effect concentrations dramatically enhances steroid hormone action. Environ. Health Perspect. 110, 917-921.

Scholze, M., Boedeker, W., Faust, M., Backhaus, T., Altenburger, R., Grimme, L.H. 2001. A general best-fit method for concentration-response curves and the estimation of low-effect concentrations. Environ. Toxicol. Chem. 20, 448-457.

Seki, M., Yokota, H., Maeda, M., Tadokoro, H., Kobayashi, K., 2003. Effects of 4 nonylphenol and 4-tert-octylphenol on sex differentiation and vitellogenin induction in medaka (Oryzias latipes). Environ. Toxicol. Chem. 22, 1507-1516.

Sheahan, D.A., Brighty, G.C., Daniel, M., Kirby, S.J., Hurst, M.R., Kennedy, J., Morris, S. Routledge, E.J., Sumpter, J.P., Waldock, M.J., 2002. Estrogenic activity measured in a sewage treatment works treating industrial inputs containing high concentrations of alkylphenolic compounds - a case study. Environ. Toxicol. Chem. 21, 507-514.

Silva, E., Rajapakse, N., Kortenkamp, A., 2002. Something from "nothing" - eight weak estrogenic chemicals combined at concentrations below NOECs produce significant mixture effects. Environ. Sci. Technol. 36, 1751-1756.
Staples, C.A., Dorn, P.B., Klecka, G.M., O’Block, S.T., Branson, D.R., Harris, L.R., 2000. Bisphenol A concentrations in receiving waters near US manufacturing and processing facilities. Chemosphere 40, 521-525.

Sullivan, K.B., Spence, K.M., 2003. Effects of sublethal concentrations of atrazine and nitrate on metamorphosis of the African clawed frog. Environ. Toxicol. Chem. 22, 627-635.

Sumpter, J.P., Jobling, S., 1995. Vitellogenesis as a biomarker for estrogenic contamination of the aquatic environment. Environ. Health Perspect. 103, 173-178.

Thompson, S., Tilton, F., Schlenk, D., Benson, W.H., 2000. Comparative vitellogenic responses in three teleost species: extrapolation to in situ field studies. Mar. Environ. Res. 50, 185-189.

Thorpe, K.L., Cummings, R.I., Hutchinson, T.H., Scholze, M., Brighty, G., Sumpter, J.P., Tyler, C.R., 2003. Relative potencies and combination effects of steroidal estrogens in fish. Environ. Sci. Technol. 37, 1142-1149.

Thorpe, K.L., Hutchinson, T.H., Hetheridge, M.J., Scholze, M., Sumpter, J.P., Tyler, C.R., 2001. Assessing the biological potency of binary mixtures of environmental estrogens using vitellogenin induction in juvenile rainbow trout (Oncorhynchus mykiss). Environ. Sci. Technol. 35, 2476-2481.

Tyler, C.R., Jobling, S.R., Sumpter, J.P., 1998. Endocrine disruption in wildlife: a critical review of the evidence. Crit. Rev. Toxicol. 28, 319-361.

US EPA, 2000. Supplementary guidance for conducting health risk assessment of chemical mixtures. Washington, DC.

US EPA, 2002. Draft detailed review paper on fish screening assays for endocrine disruption. Columbus, Ohio.

Witorsch, R.J., 2002. Endocrine disruptors: can biological effects and environmental risks be predicted? Regul. Toxicol. Pharm. 36, 118-130.

Yamaguchi, A., Ishibashi, H., Kohra, S., Arizono, K., Tominaga, N., 2005. Short-term effects of endocrine-disrupting chemicals on the expression of estrogenresponsive genes in male medaka (Oryzias latipes). Aquat. Toxicol. 72, 239-249.

Zhou, Y.Q., 2008. Source and fate of endocrine disrupting compounds in different water body in Beijing, China. Ph.D. dissertation. Chinese Academy of Sciences. 